\title{
Determination of Carnitine Ester Patterns During the Second Half of Pregnancy, at Delivery, and in Neonatal Cord Blood by Tandem Mass Spectrometry: Complex and Dynamic Involvement of Carnitine in the Intermediary Metabolism
}

\author{
GÁBOR C. TALIÁN, KATALIN KOMLÓSI, TAMÁS DECSI, BERTHOLD KOLETZKO, AND BÉLA MELEGH \\ Department of Medical Genetics and Child Development [G.C.T, K.K., B.M], Department of Pediatrics [T.D.], University of Pécs, Pécs, \\ Hungary, H-7624, Dr. von Hauner Children's Hospital [B.K.], Ludwig-Maximilians University, Munich, Germany, D-80337
}

\begin{abstract}
We studied plasma concentrations of free carnitine and 30 carnitine esters by electron spray ionization (ESI) tandem mass spectrometry in 37 pregnant women at the 20th and 30th weeks of gestation and at delivery, and in their neonates at birth, and in 22 age-matched nonpregnant women. The plasma levels of acetylcarnitine and carnitine esters with more than five carbons were significantly higher, whereas the concentration of free carnitine was significantly lower at term than at the 20th week of pregnancy (16.75 \pm 0.89 versus $19.61 \pm 1.25$ ). Almost all of $\mathrm{C} 2$ - to $\mathrm{C} 12$-carnitine esters were significantly lower, whereas $\mathrm{C} 16$ - and C18-carnitines with in-chain modifications were significantly higher in mothers at delivery compared with nonpregnant women. Plasma levels of free carnitine and C2-, C3-, C4-, C5-, C6-, and C16-carnitines were significantly lower, while concentrations of carnitine esters with $8,10,12$ and 18 carbons in the acyl chain as well as C14:1-, C14:2-, and C16:1-OH-carnitines were significantly higher in mothers at term than in their neonates. The data of the present study clearly show dynamic features of plasma carnitine profile during pregnancy and indicate an extraordinarily active participation of the carnitine in the intermediary metabolism both in the pregnant woman and in the neonate. (Pediatr Res 62: 88-92, 2007)
\end{abstract}

D uring the first two trimesters of pregnancy, the maternal metabolism continuously adapts, with accretion of fat stores deriving from aliments and then shifting to an accelerated catabolism of lipids in the late gestation (1). In human newborns, fat represents $15-16 \%$ of total body composition, and the accumulation of white adipose tissue occurs predominantly during the last trimester (2).

Carnitine is a conditionally essential quaternary amino acid that plays a crucial role in beta-oxidation of long-chain fatty acids and in ketogenesis (3-5). The carnitine molecule can form esters with FFA of different chain length; the endoge-

Received September 7, 2006; accepted February 26, 2007.

Correspondence: Béla Melegh, M.D., Ph.D., Professor of Medical Genetics and Pediatrics, Department of Medical Genetics and Child Development, University of Pécs, H-7624, Pécs, Szigeti str. 12, Hungary; e-mail: Bela.Melegh@aok.pte.hu

This study was financially supported by the Hungarian National Research Fund (OTKA T 046630 for T.D. and OTKA T 049589 for B.M.) and the Hungarian Ministry of Health (ETT 497/2006 for B.M.). The studies reported herein have been carried out with partial financial support from the Commission of the European Communities, specific RTD Programme "Quality of Life and Management of Living Resources", within the 5th Framework Programme (Contract No. QLK1-CT-1999-00888 NUHEAL [Nutraceuticals for a healthier life]). This manuscript does not necessarily reflect the views of the Commission and in no way anticipates the future policy in this area. nous (4) or exogenous acyl groups (6-8) are transferred from coenzyme-A to carnitine by different carnitine acyltransferases (9).

Availability of carnitine has been reported to be essential for the developing fetus $(10,11)$, and carnitine is stored in increasing amounts in fetal tissues, mainly in the liver and muscle, during the last period of gestation (12). Fetal carnitine is derived from the mother via transplacental transfer of carnitine (13). The $\mathrm{Na}^{+}$-dependent high-affinity carnitine transporter OCTN2 $(14,15)$ is expressed in human placenta (16), and placental OCTN2 is necessary for the accumulation of carnitine in placenta and fetus, and, consequently, for the oxidation of long-chain fatty acids in fetal-placental unit $(17,18)$. Studies on OCTN2 -/- mice suggest that the placental carnitine synthesis might depend on the carnitine status of the fetus and/or the mother (13). On the basis of these observations, it is clear that further studies of maternal and neonatal carnitine status are needed, including the investigation of participation of carnitine in the ester metabolism, as a natural extension. The primary goal of the current study was to characterize the pregnancy-related alterations of plasma carnitine esters by the use of ESI triple quadrupole tandem MS as an approach to the better understanding of the maternofetal carnitine homeostasis of humans. The study design enabled us to make a multilateral analysis of data, which included analysis of the pattern during the pregnancy and comparison of the results of mothers with their infants and with controls.

\section{MATERIALS AND METHODS}

Study groups. The mothers included into the present study participated in an international research project focusing on pregnancy-related alterations of lipid metabolism and effects of nutritional supplementation during the second half of pregnancy. Detailed description of the study has been published elsewhere (19). Briefly, inclusion criteria for pregnant women were age between 18 and $40 \mathrm{y}$ at study entry and weight between 50 and $90 \mathrm{~kg}$ at the 20th week of pregnancy. Each mother gave her informed consent to the study and filled in a detailed questionnaire; anthropometric and lifestyle information, physical status, laboratory parameters, and nutritional characteristics of the mothers were recorded throughout pregnancy (3). The circumstances of delivery and the results of physical and laboratory examinations of the newborns as well as feeding characteristics up to the 24th week were also

Abbreviations: DC, dicarboxylic acid; ESI, electron spray ionization; MS, mass spectrometer or mass spectrometry 
Table 1. Pertinent clinical and anthropometric data of mothers and their newborn babies

\begin{tabular}{lr}
\hline Mothers $(n=37)$ & \\
\hline Age at study entry $(\mathrm{Y})$ & $29.03 \pm 0.86$ \\
Height $(\mathrm{cm})$ & $164.9 \pm 0.99$ \\
Weight $(\mathrm{kg})$ & $68.77 \pm 1.99$ \\
20th week & $74.98 \pm 2.47$ \\
30th week & $78.16 \pm 2.24$ \\
Delivery & \\
BMI $\left(\mathrm{kg} / \mathrm{m}^{2}\right)$ & $25.29 \pm 0.77$ \\
20th week & $27.58 \pm 0.89$ \\
30th week & $28.75 \pm 0.80$ \\
Delivery & \\
\hline Newborns $(n=37)$ & $36.73 \pm 0.28$ \\
\hline Gestational age $(\mathrm{wk})$ & $49.5 \pm 0.44$ \\
Length $(\mathrm{cm})$ & $3.24 \pm 0.08$ \\
Weight $(\mathrm{kg})$ &
\end{tabular}

Data are mean \pm SEM.

recorded in detail. The study design has been approved by the Ethics Committee of the University of Pécs.

All deliveries were uncomplicated singleton births; 20 boys (54\%) and 17 girls $(46 \%)$ were born. The Apgar scores were all normal both at 1 min and at $5 \mathrm{~min}$. Six newborns were born premature; however, gestational ages were $\geq 35 \mathrm{wk}$ in all these babies. Anthropometric parameters of mothers and newborns are shown in Table 1.

We aimed to follow changes of carnitine profiles of pregnant women investigated at the 20th and 30th week of gestation and at term. Fasting venous blood plasma samples of 37 mothers were available for all these three time points; these data were used in the current study. We also obtained fasting blood plasma of 22 nonpregnant, apparently healthy and omnivorous women of similar age $(28.45 \pm 1.43 \mathrm{y}$, mean \pm SEM). Furthermore, venous cord blood samples from 20 newborns of the 37 mothers participating in this study were also available for carnitine profiling.

Mass spectrometry. The method used in the present study was essentially a modification of the procedure described elsewhere (20).

Sample preparation. Blood samples were drawn into tubes containing EDTA as anticoagulant and stored at $-80^{\circ} \mathrm{C}$ until analysis. Ten microliters of each plasma sample was dropped onto a filter paper (Schleicher \& Schüell 2992, Dassel, Germany) and dried for $2 \mathrm{~h}$ then the resulting spot was accurately cut off and placed into an Eppendorf-tube. Two hundred microliters methanol was added, containing $0.76 \mu \mathrm{M}{ }^{2} \mathrm{H}_{3}$-carnitine, $0.04 \mu \mathrm{M}{ }^{2} \mathrm{H}_{3}-$ propionylcarnitine, $0.04 \mu \mathrm{M}{ }^{2} \mathrm{H}_{3}$-octanoylcarnitine, and $0.08 \mu \mathrm{M}{ }^{2} \mathrm{H}_{3}$ palmitoylcarnitine as internal standards. The vials were gently shaken for 20 min and the extracts were transferred into a new tube and dried at $40^{\circ} \mathrm{C}$ under a mild nitrogen stream. One hundred microliters of i-butanol- $\mathrm{HCl}$ were pipetted into each vial and the samples were incubated for $15 \mathrm{~min}$ while shaken at $65^{\circ} \mathrm{C}$ in a hybridization oven. Excessive butanol- $\mathrm{HCl}$ was removed by evaporating at $40^{\circ} \mathrm{C}$ under nitrogen stream and carnitine ester derivatives were dissolved in $100 \mu \mathrm{L}$ acetonitrile:water (80:20 v: $\mathrm{v} \%$ ) eluent mixture. This procedure is known to result in conversion of a small quantity of C2-carnitine into free carnitine, which can lead to an artificial increase of its level (21).

MS/MS. A Waters 2795 HPLC instrument (Waters Corp., Milford, MA) was applied for the solvent delivery providing a $0.1 \mathrm{~mL} / \mathrm{min}$ stream of acetonitrile:water (80:20 v:v\%). Ten microliters of sample was injected into the flow at 4-min intervals. A Micromass Quattro Ultima triple quadrupole tandem mass spectrometer instrument (Waters Corp.) equipped with an ESI source used in positive mode was applied for analysis. For nebulization and desolvation, nitrogen gas was used, and argon served as collision gas.

The analysis method consisted of 78 complete parent scans with MS1 scanning a range of molecular masses between 200 and 550 Da, and MS3 adjusted to the fragment ion mass of $85 \mathrm{Da}$. The optimized cone voltage and collision energy were $55 \mathrm{~V}$ and $26 \mathrm{eV}$, respectively.

(Since 2003, our mass spectrometry facility is in the Newborn Screening Quality Assurance Program organized by the Centers for Disease Control and Prevention (http://www.cdc.gov/labstandards/msgap_program_background. $\mathrm{htm})$. This activity involves controlled measurements of selected acylcarnitines in two quality control regimens and four proficiency tests yearly.)

Statistics. For correlation analysis between maternal and newborn values, we used the Pearson's bivariate test; all other comparisons were made with unpaired $t$ test. Statistical analysis was performed by using the SPSS 11.5 software package (SPSS Inc., Chicago, IL).

\section{RESULTS}

Comparison of stages of pregnancy. Changes of the carnitine ester profile are shown in Table 2. The level of free carnitine decreased at the 30th week and remained lower during the further period of the pregnancy. Without significant changes in some short-chain carnitine esters, the C2-, C5:1-, C4DC-, and C5DC-carnitine and acylcarnitines containing more than five carbon atoms in their acyl chain showed a gradual increase during the last period of the gravidity, reaching the highest levels at term. The degree of the increment for these carnitine esters was greater during the last $10 \mathrm{wk}$ of pregnancy. The most pronounced increases were observed in the concentrations of C14:1-carnitine (2.24 times from the 20th week to term), C18:1- (2.19), C18:2- (2.00), C16:1(1.88), and C12:1-, C16- and C18:2-OH-carnitines (1.80). As a general consequence, the total calculated sum of the carnitine esters (called here as total acylcarnitines) also rose in the last period of the pregnancy compared with the earlier stage(s).

Comparison of mothers at term and nonpregnant women. Comparing the carnitine profiles of mothers at delivery with nonpregnant controls, almost all of $\mathrm{C} 2$ - to $\mathrm{C} 12$-carnitine ester levels were significantly lower in the pregnant women. The largest difference was seen for the $\mathrm{C} 10-(0.42$ times as much in mothers at term as in the controls), for C10:1- (0.45), C3(0.47), C8- (0.47), C5- (0.53), C5-DC- (0.55), C12-(0.61), and C3- (0.61) carnitine esters. By contrast, for longer chain length esters, like many in-chain modified $\mathrm{C} 16$ - and $\mathrm{C} 18$-carnitine esters (C16:1, C16:1OH, C16-OH, C18:2, C18:1, C18:2OH, and $\mathrm{C} 181 \mathrm{OH}$ ), the levels were significantly higher in pregnant women at birth than in the controls. The levels of some of these esters were already elevated from the earlier stage of the pregnancy (C16:1-OH, C16-OH). The level of C14-, C16-, and $\mathrm{C} 18$-carnitine did not differ between the women at term and the controls. The total calculated sum of carnitine esters (shown as acylcarnitine in Table 2) in pregnant women at terms was approximately $2 / 3$ of the controls; the level of the free carnitine was in the similar range (Table 2).

Comparison of mothers at delivery and newborns. The carnitine profile of mothers at term significantly differed from their own newborns for many esters. As for the free, C2-, C3-, C4-, C5-, C6-, and C16-carnitines, the levels in mothers were lower by about one third to one half than in the neonates. In contrast, carnitine esters of $8,10,12$, and 18 carbon atoms, as well as C14:1-, C14:2-, and C16:1-OH-carnitines were significantly higher in mothers at term than in their offspring (Table 2).

Carnitine esters in the neonates and healthy adults. In the newborns, significantly lower concentrations were found for free carnitine (0.72 times of the level of the controls), C8(0.39 times), C10:1- (0.34), C10- (0.24), C4-DC- (0.69), C5-DC- (0.53), C12:1- (0.40), C12- (0.42), C6-DC- (0.72), C14:2- (0.63), C14:1 (0.54), and C18:1- (0.79) carnitines, and for total carnitine (0.76) compared with the nonpregnant female controls. In contrast, the C16- (1.27), C16:1-OH 
Table 2. Plasma carnitine concentrations ( $\mu \mathrm{mol} / \mathrm{L})$ in pregnant mothers at different stages of gestation, in nonpregnant control women and in newborns

\begin{tabular}{|c|c|c|c|c|c|}
\hline & \multicolumn{3}{|c|}{ Pregnant mothers $(n=37)$} & \multirow[b]{2}{*}{ Controls $(n=22)$} & \multirow[b]{2}{*}{ Newborns $(n=20)$} \\
\hline & 20th week & 30th week & Term & & \\
\hline Free carnitine & $19.605 \pm 1.247 \uparrow \S$ & $16.717 \pm 0.926$ & $16.748 \pm 0.895$ & $27.897 \pm 1.417 \S$ & $19.996 \pm 1.299 \S *$ \\
\hline Acylcarnitines & $7.662 \pm 0.357 \S$ & $7.666 \pm 0.592 \S$ & $10.417 \pm 0.537$ & $16.037 \pm 0.883 \S$ & $13.187 \pm 1.541 \S$ \\
\hline Total carnitine & $27.267 \pm 1.464 \dagger$ & $24.383 \pm 1.349 \S$ & $27.165 \pm 1.197$ & $43.934 \pm 2.116 \S$ & $33.184 \pm 2.587 \S *$ \\
\hline C2-carnitine & $6.237 \pm 0.318 \S$ & $6.299 \pm 0.562 \S$ & $8.482 \pm 0.494$ & $13.755 \pm 0.805 \S$ & $11.241 \pm 1.555 \S$ \\
\hline C3-carnitine & $0.112 \pm 0.008 \dagger$ & $0.098 \pm 0.005$ & $0.100 \pm 0.005$ & $0.204 \pm 0.013 \S$ & $0.224 \pm 0.015 \S$ \\
\hline C4-carnitine & $0.260 \pm 0.014$ & $0.244 \pm 0.014$ & $0.241 \pm 0.012$ & $0.335 \pm 0.015 \S$ & $0.324 \pm 0.020 \S$ \\
\hline C5:1-carnitine & $0.035 \pm 0.002 \S$ & $0.036 \pm 0.003$ & $0.041 \pm 0.004$ & $0.040 \pm 0.003$ & $0.034 \pm 0.003$ \\
\hline C5-carnitine & $0.068 \pm 0.004$ & $0.067 \pm 0.004$ & $0.074 \pm 0.005$ & $0.139 \pm 0.009 \S$ & $0.140 \pm 0.017 \S$ \\
\hline C6-carnitine & $0.059 \pm 0.003 \uparrow \S$ & $0.067 \pm 0.004$ & $0.072 \pm 0.003$ & $0.099 \pm 0.006 \S$ & $0.108 \pm 0.007 \S$ \\
\hline C5-OH-carnitine & $0.023 \pm 0.001$ & $0.024 \pm 0.001$ & $0.024 \pm 0.001$ & $0.035 \pm 0.002 \S$ & $0.033 \pm 0.003 \S$ \\
\hline C8:1-carnitine & $0.062 \pm 0.006 \S$ & $0.069 \pm 0.004 \S$ & $0.077 \pm 0.005$ & $0.083 \pm 0.007$ & $0.078 \pm 0.012$ \\
\hline C8-carnitine & $0.048 \pm 0.003 \S$ & $0.054 \pm 0.004 \S$ & $0.065 \pm 0.004$ & $0.130 \pm 0.009 \S$ & $0.050 \pm 0.004 \S *$ \\
\hline C10:2-carnitine & $0.016 \pm 0.001 \uparrow \S$ & $0.019 \pm 0.001$ & $0.021 \pm 0.001$ & $0.017 \pm 0.001 \S$ & $0.019 \pm 0.001$ \\
\hline C10:1-carnitine & $0.043 \pm 0.003 \S$ & $0.049 \pm 0.003 \S$ & $0.058 \pm 0.003$ & $0.126 \pm 0.008 \S$ & $0.043 \pm 0.004 \S *$ \\
\hline C10-carnitine & $0.050 \pm 0.004 \S$ & $0.058 \pm 0.006 \S$ & $0.068 \pm 0.004$ & $0.152 \pm 0.012 \S$ & $0.036 \pm 0.003 \S *$ \\
\hline C4-DC-carnitine & $0.024 \pm 0.002 \S$ & $0.026 \pm 0.001 \S$ & $0.030 \pm 0.002$ & $0.047 \pm 0.003 \S$ & $0.033 \pm 0.007 *$ \\
\hline C5-DC-carnitine & $0.020 \pm 0.001 \S$ & $0.020 \pm 0.001 \S$ & $0.025 \pm 0.001$ & $0.046 \pm 0.002 \S$ & $0.024 \pm 0.002 *$ \\
\hline C12:1-carnitine & $0.018 \pm 0.001 \S$ & $0.019 \pm 0.002 \S$ & $0.032 \pm 0.003$ & $0.050 \pm 0.004 \S$ & $0.020 \pm 0.002 \S *$ \\
\hline C12-carnitine & $0.019 \pm 0.001 \S$ & $0.018 \pm 0.001 \S$ & $0.032 \pm 0.002$ & $0.050 \pm 0.004 \S$ & $0.021 \pm 0.002 \S *$ \\
\hline C6-DC-carnitine & $0.014 \pm 0.001 \S$ & $0.015 \pm 0.001 \S$ & $0.021 \pm 0.002$ & $0.025 \pm 0.002$ & $0.018 \pm 0.002 *$ \\
\hline C14:2-carnitine & $0.014 \pm 0.001 \S$ & $0.014 \pm 0.001 \S$ & $0.024 \pm 0.002$ & $0.028 \pm 0.003$ & $0.018 \pm 0.002 \S *$ \\
\hline C14:1-carnitine & $0.017 \pm 0.001 \S$ & $0.019 \pm 0.001 \S$ & $0.037 \pm 0.004$ & $0.040 \pm 0.004$ & $0.022 \pm 0.002 \S *$ \\
\hline C14-carnitine & $0.016 \pm 0.001 \S$ & $0.016 \pm 0.001 \S$ & $0.026 \pm 0.002$ & $0.024 \pm 0.002$ & $0.024 \pm 0.002$ \\
\hline C8-DC-carnitine & $0.011 \pm 0.001 \S$ & $0.010 \pm 0.001 \S$ & $0.016 \pm 0.001$ & $0.012 \pm 0.001 \S$ & $0.017 \pm 0.003$ \\
\hline C16:1-carnitine & $0.027 \pm 0.002 \S$ & $0.028 \pm 0.002 \S$ & $0.052 \pm 0.004$ & $0.039 \pm 0.003 \S$ & $0.047 \pm 0.010$ \\
\hline C16-carnitine & $0.071 \pm 0.004 \S$ & $0.065 \pm 0.004 \S$ & $0.127 \pm 0.007$ & $0.120 \pm 0.008$ & $0.152 \pm 0.013 \S *$ \\
\hline C16:1-OH-carnitine & $0.046 \pm 0.003 \S$ & $0.048 \pm 0.003 \S$ & $0.056 \pm 0.002$ & $0.028 \pm 0.002 \S$ & $0.046 \pm 0.003 \S *$ \\
\hline C16-OH-carnitine & $0.045 \pm 0.002$ & $0.044 \pm 0.003 \S$ & $0.051 \pm 0.003$ & $0.023 \pm 0.002 \S$ & $0.047 \pm 0.004 *$ \\
\hline C18:2-carnitine & $0.082 \pm 0.005 \S$ & $0.080 \pm 0.004 \S$ & $0.164 \pm 0.010$ & $0.107 \pm 0.009 \S$ & $0.124 \pm 0.012 \S$ \\
\hline C18:1-carnitine & $0.110 \pm 0.007 \S$ & $0.106 \pm 0.007 \S$ & $0.242 \pm 0.014$ & $0.154 \pm 0.013 \S$ & $0.121 \pm 0.010 \S *$ \\
\hline C18-carnitine & $0.065 \pm 0.005 \uparrow \S$ & $0.050 \pm 0.003 \S$ & $0.088 \pm 0.005$ & $0.078 \pm 0.006$ & $0.071 \pm 0.007 \S$ \\
\hline C18:2-OH-carnitine & $0.018 \pm 0.002 \S$ & $0.019 \pm 0.001 \S$ & $0.033 \pm 0.003$ & $0.019 \pm 0.002 \S$ & $0.024 \pm 0.003 \S$ \\
\hline C18:1-OH-carnitine & $0.019 \pm 0.001 \S$ & $0.019 \pm 0.002 \S$ & $0.027 \pm 0.003$ & $0.014 \pm 0.001 \S$ & $0.018 \pm 0.002 \S$ \\
\hline
\end{tabular}

Data are mean \pm SEM. Values marked with different symbols are statistically significant.

$* p<0.05$ vs controls.

$\dagger p<0.05$ vs 30th week.

$\S p<0.05 v s$ at term.

(1.62), and $\mathrm{C} 16-\mathrm{OH}(2.00)$ levels were significantly higher in the infants (Table 2).

Correlation between maternal and newborn profiles. We calculated the correlation between the corresponding values of the carnitine profile of the mothers at term and their own newborns. Significant positive correlations were seen for the free carnitine, C6-DC-, C8-DC-, C8:1-, C10:2-, C18:1-, and C18:2-carnitine esters (Table 3).

\section{DISCUSSION}

The design of the present study enabled us to analyze the blood plasma carnitine esters 1) during the second half of the pregnancy; 2) to compare the individual esters in pregnant and nonpregnant women; 3 ) measure the carnitine esters in neonatal cord blood and compare them with the mothers and with the nonpregnant women; and 4) to search for possible correlations between carnitine ester levels of mothers and their newborns. We used the ESI triple quadrupole tandem MS, which allowed a precise and accurate determination of 30 carnitine esters besides the circulating free carnitine. Similar trial has not been presented in the literature so far, and the ESI/MS/MS based profiling presented here revealed very complex and dynamic changes in circulating acylcarnitines.

During the second part of the pregnancy, we observed a decreased level of the free carnitine and carnitine esters, which was already documented also by others, who used the classic radioenzymatic free carnitine assays (22-25). By contrast, the coupled alkaline hydrolysis based determination methods, which was used in the previous decades of the carnitine research, could not provide information on the individual carnitine esters. In the present study, we observed an increase for the majority of carnitine ester levels, which led to an elevation in the total calculated amount of carnitine esters. Specifically, we observed significant rise in acetylcarnitine and carnitine esters with more than five carbons. The highest increases were seen for most of the C12-C18 carnitines, which may reflect the enhanced fatty acid oxidation in maternal tissues and correlates well with the elevated FFA levels in the mother's circulation during late gestation (26,27). Only moderate or no changes were seen for the short-chain 
Table 3. Correlation analysis between carnitine profiles of mother-infant pairs at term $(n=20)$

\begin{tabular}{|c|c|c|}
\hline & $\begin{array}{c}\text { Correlation } \\
\text { coefficient }\end{array}$ & $p$ \\
\hline Free carnitine & 0.645 & $0.007 \S$ \\
\hline Acylcarnitines & -0.014 & $0.958^{\circ}$ \\
\hline Total carnitine & 0.344 & 0.192 \\
\hline C2-carnitine & -0.003 & 0.992 \\
\hline C3-carnitine & 0.429 & 0.097 \\
\hline C4-carnitine & 0.283 & 0.289 \\
\hline C5:1-carnitine & 0.418 & 0.107 \\
\hline C5-carnitine & 0.003 & 0.991 \\
\hline C6-carnitine & 0.166 & 0.539 \\
\hline C5-OH-carnitine & 0.273 & 0.306 \\
\hline C8:1-carnitine & 0.529 & $0.035 \S$ \\
\hline C8-carnitine & 0.319 & 0.228 \\
\hline C10:2-carnitine & 0.689 & $0.003 \S$ \\
\hline C10:1-carnitine & -0.190 & 0.482 \\
\hline C10-carnitine & -0.109 & 0.687 \\
\hline C4-DC-carnitine & 0.139 & 0.608 \\
\hline C5-DC-carnitine & -0.018 & 0.947 \\
\hline C12:1-carnitine & 0.429 & 0.097 \\
\hline C12-carnitine & 0.232 & 0.388 \\
\hline C6-DC-carnitine & 0.687 & $0.003 \S$ \\
\hline C14:2-carnitine & 0.058 & 0.830 \\
\hline C14:1-carnitine & 0.355 & 0.178 \\
\hline C14-carnitine & 0.276 & 0.300 \\
\hline C8-DC-carnitine & 0.771 & $0.001 \S$ \\
\hline C16:1-carnitine & 0.068 & 0.802 \\
\hline C16-carnitine & 0.404 & 0.120 \\
\hline C16:1-OH-carnitine & 0.025 & 0.928 \\
\hline C16-OH-carnitine & 0.173 & 0.522 \\
\hline C18:2-carnitine & 0.596 & $0.015 \S$ \\
\hline C18:1-carnitine & 0.598 & $0.014 \S$ \\
\hline C18-carnitine & 0.067 & 0.805 \\
\hline C18:2-OH-carnitine & 0.271 & 0.310 \\
\hline C18:1-OH-carnitine & 0.393 & 0.132 \\
\hline
\end{tabular}

$\S p<0.05$.

carnitine esters, which are derived mainly from amino acid catabolism (28).

Comparing the pregnant with the nonpregnant women revealed unique features that had not been detected in relation to any metabolic condition, therefore suggesting a gravidityspecific, dynamic modification of the carnitine ester metabolism. Almost all of C2- to C12-carnitine ester levels (Table 2) were significantly lower in pregnant women at each time period of their gravidity compared with the nonpregnant controls, with the largest difference for C3-, C5-, C8-, C10- C10:1, and C12esters. By contrast, for longer chains, like the in-chain modified C16- and C18-carnitine esters, the plasma levels were significantly higher in pregnant women at birth than in the controls; the levels of some esters were already elevated from the earlier stages of the pregnancy (e.g. C16:1-OH, C16-OH). This distribution also may represent a shift in the balance of the carnitine ester homeostasis and may reflect the enhanced participation of the carnitine in the fatty acid oxidation process. Another explanation is that the decrease in free carnitine level may reflect a decrease of the total body carnitine pool in the pregnant women. For example, a greater fetal need for carnitine via the placental supply can also contribute to the decrease of the reserves.

After birth, the availability of carnitine is crucial for the infant when lipid breakdown becomes the major source of energy with switching from the oxidation of glucose to that of fatty acids (29). It is well established that the fetus has limited ability to synthesize carnitine: during total parenteral nutrition, plasma carnitine decreases in premature infants and tissue accretion of carnitine declines (30). In the cord blood, the entire carnitine ester spectrum could be detected. Our data do not differ from observations of others in respect to the levels of the individual carnitine esters (31). The long-chain acylcarnitine levels were generally lower in the cord blood as compared with the mothers (C16- and C18-carnitines), which may correlate with a more active fatty acid catabolism of the long-chain fatty acids in the mothers than in the neonates. The free carnitine in the cord blood was lower than in the nonpregnant women; this observation is in accordance with the previous data of others (23-25). Meanwhile, the free, C2-, total acyl-, and total carnitine (sum of free carnitine and all carnitine esters) concentrations in the newborns were between the maternal and the nonpregnant control levels. Mediumchain carnitine levels (C8-C14) were generally lower, in turn, C16-carnitine and its derivatives were higher in newborns than in nonpregnant women. Therefore, the carnitine ester profile in the neonates represents a unique spectrum, which differs either from the mothers or from the nonpregnant fertile women. The difference clearly reflects differential carnitine ester metabolism, however, the exact explanation is not know.

A common thought suggests that the carnitine and its derivatives do not remain compartmentalized in the placenta, or in the maternal or fetal tissues. Besides the maternofetal transport, they may regurgitate into the maternal blood stream. These aspects have not been investigated. The test for correlation between the maternal and neonatal carnitine ester concentrations also revealed specific features. Significant positive correlation was found between the maternal and neonatal levels of free carnitine, C6-DC-, C8-DC-, C10:2-, C18:1-, and C18:2-carnitine esters (Table 3). The positive correlation for these esters suggests that their transport might be influenced by their concentrations on both sides of the placenta. The absence of the correlation for majority of the esters likely reflect the existence of concentration-independent, carriermediated transport mechanisms for those esters, or their active participation in the placental metabolism. In general, the placental transport of carnitine and carnitine esters is carrier mediated. The involvement of the OCTN2 $\mathrm{Na}^{+}$-dependent, high-affinity carnitine transporter and expression of the ATB $(0,+)$ amino acid transporter with broad substrate specificity are documented $(16,32)$.

There are several possible extensions of the present study. The contribution of maternal and fetal tissues, and of placenta to the ester flux under normal and pathologic conditions remains to be investigated. Such issue can have even therapeutic consequences of possible use of carnitine administra- 
tion for example in acute fatty liver associated pregnancies or in woman who carry a fetus with a mitochondrial defect or more specifically with fatty acid oxidation disorders.

Acknowledgments. The authors thank Ilona Szántó for her help and technical skills in mass spectrometry.

\section{REFERENCES}

1. King JC, Butte NF, Bronstein MN, Kopp LE, Lindquist SA Energy metabolism during pregnancy: influence of maternal energy status. Am J Clin Nutr 1994;59:439S-445S

2. Hahn P, Novak M 1975 Development of brown and white adipose tissue. J Lipid Res 16:79-91

3. Foster DW 2004 The role of the carnitine system in human metabolism. Ann N Y Acad Sci 1033:1-16

4. Bieber LL Carnitine. Annu Rev Biochem 1988;57:261-283

5. Steiber A, Kerner J, Hoppel CL 2004 Carnitine: a nutritional, biosynthetic, and functional perspective. Mol Aspects Med 25:455-473

6. Brass EP 2002 Pivalate-generating prodrugs and carnitine homeostasis in man. Pharmacol Rev 54:589-598

7. Brass EP, Mayer MD, Mulford DJ, Stickler TK, Hoppel CL 2003 Impact on carnitine homeostasis of short-term treatment with the pivalate prodrug cefditoren pivoxil. Clin Pharmacol Ther 73:338-347

8. Melegh B, Kerner J, Bieber LL 1987 Pivampicillin-promoted excretion of pivaloylcarnitine in humans. Biochem Pharmacol 36:3405-3409

9. Jogl G, Hsiao YS, Tong L 2004 Structure and function of carnitine acyltransferases. Ann N Y Acad Sci 1033:17-29

10. Melegh B, Hermann R, Bock I 1996 Generation of hydroxytrimethyllysine from trimethyllysine limits the carnitine biosynthesis in premature infants. Acta Paediatr $85: 345-350$

11. Vaz FM, Wanders RJ 2002 Carnitine biosynthesis in mammals. Biochem J 361:417429

12. Nakano C, Takashima S, Takeshita K 1989 Carnitine concentration during the development of human tissues. Early Hum Dev 19:21-27

13. Shekhawat PS, Yang HS, Bennett MJ, Carter AL, Matern D, Tamai I, Ganapathy V 2004 Carnitine content and expression of mitochondrial beta-oxidation enzymes in placentas of wild-type $($ OCTN2 $(+/+))$ and OCTN2 Null $($ OCTN2 $(-/-))$ Mice. Pediatr Res 56:323-328

14. Tamai I, Ohashi R, Nezu J, Yabuuchi H, Oku A, Shimane M, Sai Y, Tsuji A 1998 Molecular and functional identification of sodium ion-dependent, high affinity human carnitine transporter OCTN2. J Biol Chem 273:20378-20382

15. Wu X, Prasad PD, Leibach FH, Ganapathy V 1998 cDNA sequence, transport function, and genomic organization of human OCTN2, a new member of the organic cation transporter family. Biochem Biophys Res Commun 246:589-595
16. Grube M, Schwabedissen HM, Draber K, Prager D, Moritz KU, Linnemann K, Fusch C, Jedlitschky G, Kroemer HK 2005 Expression, localization, and function of the carnitine transporter octn2 (slc22a5) in human placenta. Drug Metab Dispos $33: 31-37$

17. Rakheja D, Bennett MJ, Foster BM, Domiati-Saad R, Rogers BB 2002 Evidence for fatty acid oxidation in human placenta, and the relationship of fatty acid oxidation enzyme activities with gestational age. Placenta 23:447-450

18. Shekhawat P, Bennett MJ, Sadovsky Y, Nelson DM, Rakheja D, Strauss AW 2003 Human placenta metabolizes fatty acids: implications for fetal fatty acid oxidation disorders and maternal liver diseases. Am J Physiol Endocrinol Metab 284:E1098E1105

19. Decsi T, Campoy C, Koletzko B 2005 Effect of N-3 polyunsaturated fatty acid supplementation in pregnancy: the Nuheal trial. Adv Exp Med Biol 569:109-113

20. Bene J, Komlosi K, Havasi V, Talian G, Gasztonyi B, Horvath K, Mozsik G, Hunyady B, Melegh B, Figler M 2006 Changes of plasma fasting carnitine ester profile in patients with ulcerative colitis. World J Gastroenterol 12:110-113

21. Schulze A, Schmidt C, Kohlmuller D, Hoffmann GF, Mayatepek E 2003 Accurate measurement of free carnitine in dried blood spots by isotope-dilution electrospray tandem mass spectrometry without butylation. Clin Chim Acta 335:137-145

22. Hromadova M, Parrak V, Huttova M, Danova K, Urvolgyova M 1994 Carnitine level and several lipid parameters in venous blood of newborns, cord blood and maternal blood and milk. Endocr Regul 28:47-52

23. Koumantakis E, Sifakis S, Koumantaki Y, Hassan E, Matalliotakis I, Papadopoulou E, Evageliou A 2001 Plasma carnitine levels of pregnant adolescents in labor. J Pediatr Adolesc Gynecol 14:65-69

24. Novak M, Monkus EF, Chung D, Buch M 1981 Carnitine in the perinatal metabolism of lipids. I. Relationship between maternal and fetal plasma levels of carnitine and acylcarnitines. Pediatrics 67:95-100

25. Winter SC, Linn LS, Helton E 1995 Plasma carnitine concentrations in pregnancy, cord blood, and neonates and children. Clin Chim Acta 243:87-93

26. Herrera E, Amusquivar E 2000 Lipid metabolism in the fetus and the newborn. Diabetes Metab Res Rev 16:202-210

27. Herrera E 2000 Metabolic adaptations in pregnancy and their implications for the availability of substrates to the fetus. Eur J Clin Nutr 54:S47-S51

28. Costa CC, de Almeida IT, Jakobs C, Poll-The BT, Duran M 1999 Dynamic changes of plasma acylcarnitine levels induced by fasting and sunflower oil challenge test in children. Pediatr Res 46:440-444

29. Wolf H, Stave U, Novak M, Monkus EF 1974 Recent investigations on neonatal fat metabolism. J Perinat Med 2:75-87

30. Schmidt-Sommerfeld E, Penn D 1990 Carnitine and total parenteral nutrition of the neonate. Biol Neonate 58:81-88

31. Meyburg J, Schulze A, Kohlmueller D, Linderkamp O, Mayatepek E 2001 Postnatal changes in neonatal acylcarnitine profile. Pediatr Res 49:125-129

32. Kekuda R, Prasad PD, Fei YJ, Torres-Zamorano V, Sinha S, Yang-Feng TL, Leibach FH, Ganapathy V 1996 Cloning of the sodium-dependent, broad-scope, neutral amino acid transporter Bo from a human placental choriocarcinoma cell line. J Biol Chem 271:18657-18661 